\title{
Review of Suppes 1957 Proposals For Division by Zero
}

\author{
James A.D.W. Anderson \\ james.a.d.w.anderson@btinternet.com \\ 88 Lower Henley Road, Caversham, Reading, England, RG4 5LE
}

\author{
Jan A. Bergstra \\ j.a.bergstra@uva.nl,janaldertb@gmail.com \\ Informatics Institute, University of Amsterdam, \\ Science Park 904, 1098 XH, Amsterdam, The Netherlands
}

Submitted: 26 April 2020

Revised: 8 June 2021

\begin{abstract}
We review the exposition of division by zero and the definition of total arithmetical functions in "Introduction to Logic" by Patrick Suppes, 1957, and provide a hyperlink to the archived text. This book is a pedagogical introduction to first-order predicate calculus with logical, mathematical, physical and philosophical examples, some presented in exercises. It is notable for (i) presenting division by zero as a problem worthy of contemplation, (ii) considering five totalisations of real arithmetic, and (iii) making the observation that each of these solutions to "the problem of division by zero" has both advantages and disadvantages - none of the proposals being fully satisfactory.

We classify totalisations by the number of non-real symbols they introduce, called their Extension Type. We compare Suppes' proposals for division by zero to more recent proposals. We find that all totalisations of Extension Type 0 are arbitrary, hence all non-arbitrary totalisations are of Extension Type at least 1. Totalisations of the differential and integral calculus have Extension Type at least 2. In particular, Meadows have Extension Type 1, Wheels have Extension Type 2, and Transreal numbers have Extension Type 3.

It appears that Suppes was the modern originator of the idea that all real numbers divided by zero are equal to zero. This has Extension Type 0 and is, therefore, arbitrary.
\end{abstract}




\section{Introduction}

We review Chapter 8 of Introduction to Logic by Patrick Suppes, 1957 [11] (referred to below as Suppes 1957), with a focus on Sections 8.5. and 8.7. This book is a pedagogical introduction to first-order predicate calculus with logical, mathematical, philosophical and physical examples, including an axiomatisation of Newton's Laws of Motion. Some examples are presented in exercises, though the exercises do not have solutions. The book has some historical merit in that it was written during a very productive period in the development of set theory but modern pedagogical texts are more approachable and give more support in terms of worked examples. Our main interest in reviewing chapter 8, "Theory of Definition," is that it presents the logical foundations of real arithmetic and considers five logical ways to totalise real arithmetic by allowing division by zero. These are of both historical and technical interest.

To the best of our knowledge, Suppes 1957 is the first among modern mathematicians to highlight division by zero as a problem worthy of attention but see [2] for some pre-modern and ancient views on division by zero. Suppes proposes several different solutions to the problem of division by zero but concludes that none of them is "uniformly satisfactory" (Suppes 1957 p. 164). This invites continuing research to find satisfactory solutions.

We introduce a classification of totalisations of rational or real arithmetic in terms of the number of non-real elements that are introduced, where it is assumed that such elements cannot be considered elements of known field extensions of the reals. This number of additional elements is referred to as the extension size. A totalisation of arithmetic with extension size $k$ is also referred to as having Extension Type $k$. A totalisation with extension size 0 introduces no non-real symbols. For example, the totalisation adopting $x / 0=0$ is Extension Type 0 because the quotient, the right most symbol, is a real number, zero.

The totalisation $x / 0=\perp$, with $\perp$ an absorptive element, has Extension Type 1 because $\perp$ is not a real number. For this approach we refer to the common meadows as discussed, for example, in [3].

Some totalisations have more than one Extension Type. For example, the compilation of computer languages employs epsilon $(\epsilon)$ productions which use the symbol $\epsilon$ in the compiler to correspond to the absence of a symbol in the compiled language. See, for example, [9]. This gives rise to what we now call epsilon totalisations. Consider the function $f(x)=y$. This function is total if and only if for every $x_{i}$ there exists a $y_{i}$ but it is partial if there exists an $x_{i}$ for which there is no $y_{i}$. A partial function can be totalised by writing $f\left(x_{i}\right)=\epsilon$. Here " $f\left(x_{i}\right)=\epsilon$ " is an Extension Type 1 grammatical production that adds the Greek letter, $\epsilon$, to the real numbers but it compiles to the sentence " $f\left(x_{i}\right)=$ " which is partial and, in this case, degenerate. As the compiled sentence does not add any symbols to the real numbers, it has Extension Type 0. Put another way, the extension type of a sentence and its corresponding meta sentence can be different.

Real analysis extends the real numbers by adopting two non-real symbols: $-\infty, \infty$. Thus real analysis is Extension Type 2. Wheels [4] are 
an Extension Type 2 totalisation because they add two non-real symbols: $\infty, \perp$. Transreal arithmetic (see, for example, [1], [5]) is a Type 3 totalisation because it adds three non-real symbols: $-\infty, \infty, \Phi$.

Suppes 1957 contemplates a Type $\aleph_{0}$ totalisation that takes $x / 0=$ $s$, where $s$ is a sentence of first-order predicate calculus that defines an element. This discrete language has as many sentences, $s$, as there are natural numbers so it has cardinality $\aleph_{0}$. Suppes 1957 provides no further details on this idea but observes that $s \neq s$, which leads to implausible conclusions!

\subsection{The Problem of Division by Zero}

Suppes 1957 uses The Problem of Division by Zero as the title for his Section 8.5. No reference is made to any previous work on division by zero. The problem is explained in such a manner that it extends to all partial functions on numbers. In his Section 8.5, Suppes considers how the occurrence of a zero divisor complicates the definition of division. That is, in his Section 8.5, Suppes' Problem of Division by Zero is not so much how to define division by zero, it is how to define general division so that it does not allow division by zero.

\subsection{Five Approaches}

In his Section 8.7, Five Approaches to Division by Zero, Suppes 1957 discusses and subsequently rejects the option to change logic in such a manner that $1 / 0=1 / 0$ need not be given a meaning.

The second option is that for all real $x, 1 / x$ is chosen to be some real number. Suppes rejects this option because it is wholly uninformative about simple questions, such as, is $1 / 0=2 / 0$ ?

The third option is, for each $x, x / 0=0$. Suppes considers this option is feasible but has the disadvantage that it is unfamiliar to mathematicians - a judgement which is valid around 2020, just as it was around 1960 .

The fourth option is to be explicit about the idea that for each real number $x, x / 0$ lies outside the set of real numbers. Suppes says that, in order to avoid a proliferation of elements, it is plausible to assume that, for all real $x$ and $y, x / 0=y / 0$. Implicitly this remark indicates the possibility of adopting different options for introducing and using new values outside the well-known number systems.

The fifth approach to division by zero is simply to forget about division as a function and to work with its graph, that is, to work with a relational calculus instead. From a logical perspective the fifth approach solves all problems, but Suppes acknowledges that it is extraordinarily awkward to make exclusive use of relations in practice, a point of view which, we think, would still be supported by most mathematicians.

\section{Review}

We now review totalisations of real arithmetic as put forward in Suppes 1957. These all occur in his Chapter 8 Theory of Definition, where Suppes 
considers the logical criteria that definitions must satisfy. The whole of this chapter is relevant to defining division by zero but we concentrate on just three sections. We review these difficult cases in our Subsection 2.1 The Problem of Division by Zero. In his Section 8.6 Conditional Definitions, Suppes considers the usual conditional definitions used in the mathematics of his day but points out their shortcoming. We review Suppes' argument in our Subsection 2.2 Conditional Definitions. In his Section 8.7 Five Approaches to Division by Zero, Suppes considers five totalisations of division by zero. We consider these totalisations grouped by the totalisation type we introduced above.

After the review, we discuss implications for modern research and the public understanding of science. Finally, we draw a few salient conclusions from our review and discussion.

\subsection{The Problem of defining division}

Suppes 1957 first contemplates three different logical definitions of division. Suppes' first definition of division is: $x / y=z$ if and only if $x=y \times z$. But Suppes requires that $z$ is uniquely defined for all $x, y$. This fails for two independent reasons. Firstly $z$ is not defined uniquely in $1=0 \times z$ because there is no such $z$. Secondly $z$ is not defined uniquely in $0=0 \times z$ because this equation is satisfied by all $z$. Therefore this definition is inadequate.

Suppes' second definition of division is: $x / y=z$ if and only if $y \neq 0$ and $x=y \times z$. But given an arbitrary $x$ and $y=0$, then $z$ is not defined uniquely because the definition does not say anything at all about the value of $z$. Therefore this definition is inadequate.

Suppes' third definition of division is to take his second definition but relax the requirement of requiring exactly one $z$ to requiring no more than one $z$. But this introduces two contradictions. We now present Suppes' contradictions but change variable names to make the argument clearer. It is a logical truth that $1 / 0=1 / 0$ because the left- and right-hand expressions are identical. Then, firstly, there is an $x / y=z$ with $x=1$, $y=0, z=1 / 0$. As $z$ exists, it must satisfy $x=y \times z$, but now $y \neq 0$ gives $0 \neq 0$, which is a contradiction. Secondly $x=y \times z$ gives $1=0 \times z$, which is a contradiction. Therefore this definition is inadequate.

In his Section 8.5, Suppes requires that $1 / 0$ denotes some element, so it ought to have some meaning. This leads Suppes to contemplate totalisations of division in his Section 8.7, where five perspectives on totalisation are provided.

\subsection{Conditional Definitions}

Conditional definitions begin with a hypothesis and continue with a definition that holds only in the hypothesised circumstances. Suppes defines division as: if $y \neq 0$ then $x / y=z$ if and only if $x=y \times z$. This is an arithmetically adequate definition, because it defines all cases of division that exclude division by zero, but it is logically inadequate. Definitions are supposed to allow the elimination of all of the defined symbols but we 
cannot eliminate the division symbol, /, in $1 / 0=1 / 0$ because $y=0$ and the definition does not apply in this case - by hypothesis!

Note that Suppes has now presented an arithmetically adequate but logically inadequate definition of division.

\subsection{Extension Type 0 Totalisation}

Suppes' second approach to the Problem of Division by Zero is to assert that $x / 0$ is a real number but to define division conditionally: if $y \neq 0$ then $x / y=z$ if and only if $x=y \times z$. Thus we are in the nice position that $x / 0$ is some indefinite real number, drawn from the continuum of real numbers, but we may not perform the operation of dividing by zero. As the quotient is a single real number, no symbols are added to the real numbers, making this a Type 0 totalisation.

Suppes notes that this second solution cannot decide the truth or falsity of $1 / 0=2 / 0$. We explain that this is because the left and right hand sides are indefinite real numbers, $x=1 / 0$ and $y=2 / 0$, which may be equal, $x=y$, or unequal, $x \neq y$. Now Suppes raises a remarkable objection: "The ability to prove or disprove $[1 / 0=2 / 0]$ is an argument against the second approach, since we want our axioms to be as complete as possible". This is remarkable because Suppes is saying that he wants totality.

Suppes' third solution to the Problem of Division by Zero is to define $x / 0=0$ for all real $x$. As the quotient, zero, is a real number, this is a Type 0 totalisation. This third approach avoids the undecidability of the second approach but Suppes says "many mathematicians feel uneasy with the identity: $\mathrm{x} / 0=0$ " so it is not likely to be adopted. However, some modern mathematicians do adopt this position, for example [10].

\subsection{A Radical Approach}

Suppes' fifth solution to the Problem of Division by Zero is radical. He abolishes all arithmetical operators, including division, and he abolishes all constants, such as zero, and replaces them with predicates. Thus we immediately see that this solution is arithmetically inadequate because it has eliminated the whole of arithmetic! Firstly he introduces a predicate that recognises zero: $Z(z)$ is True, if and only if $x$ is an identity element of addition, such that for all $y$, it is the case that $x+y=y$. Secondly he introduces a predicate that recognises multiplication: $M(x, y, z)$ is True if and only if $x \times y=z$. Finally he introduces a predicate that recognises division: $D(x, y, z)$ is True if and only if $\neg Z(y) \& M(y, z, x)$. There are no logical difficulties with this method of defining division - though working with predicates that recognise arithmetic is a lot more cumbersome than working with operators that perform arithmetic. When $y=0$ it is the case that $D(x, y, z)$ is False. Thus Suppes simultaneously defines general division, outlaws a continuum of cases of division by zero, and totalises division so that division by zero is false. This is a Type 0 totalisation but, while it is logically satisfactory, it is arithmetically inadequate because it has eliminated arithmetic and replaced it with a meta-description of arithmetic. 


\subsection{Extension Type 1 Totalisation}

Suppes's fourth solution to the Problem of Division by Zero is to assert that, for all real numbers $x, x / 0$ is not a real number. Furthermore, Suppes specifies that, for all real numbers $x$, there is only one $v$ such that $v=x / 0$. That is, there is exactly one non-real symbol, $v$, which makes this a Type 1 totalisation. Suppes' $v$ is an inchoate version of the modern bottom or falsum, $\perp$. But, for Suppes, it is undecidable whether $v / v$ is or is not real, whereas modern authors add sufficient axioms to totalise arithmetic over $\perp$ and specifically assert $\perp / \perp=\perp$. See, for example, [4] [3].

Suppes asserts that taking $x / 0$ non-real, "... would seem to be in closest accord with ordinary mathematical usage." The force of this assertion is that his third solution, $x / 0=0$, is not in closest accord with ordinary mathematical usage.

\subsection{Type $\aleph_{0}$ Totalisation}

Suppes' first solution to the Problem of Division by Zero is not logical but metalogical. He proposes changing first-order predicate calculus to deny meaning to expressions like $1 / 0=1 / 0$. He does not elaborate on how meaning could be denied, as against denying Truth, but he concludes that this approach has so many problems that it should be abandoned.

Notwithstanding the fact that Suppes abandons this approach, the approach exists so we must ascribe it a totalisation type. Let us be clear what Suppes outlaws and what he allows. He does not outlaw any expression or symbol, he outlaws only the meaning attached to expressions. But that is to say he does allow expressions that cannot be evaluated in real arithmetic. He explicitly allows the expression $1 / 0=1 / 0$ and invites us to allow expressions like this one. If we consider expressions to be compound or molecular symbols then, as he allows real arithmetic, he allows as many symbols of the form $(1 \times x) / 0=(1 \times x) / 0$ as can be written in first-order predicate calculus. The real numbers have cardinality the continuum, C, but only $\aleph_{0}$ of these can be written in a discrete language, such as first-order predicate calculus. Therefore we regard this approach as a totalisation with Extension Type $\aleph_{0}$.

\section{Discussion}

Suppes fourth solution to the Problem of Division by Zero, in our Subsection 2.5 Type 1 Totalisation, introduces an inchoate error term, $v$, where modern practice would use a totalised error term, $\perp$. Suppes does not find the transreal totalisation. After discarding some approaches, Suppes arrives at the position where he has three well worked out proposals for division. Suppes' conditional definition, in our Subsection 2.2 Conditional Definitions, is arithmetically satisfactory but logically unsatisfactory. Suppes fifth solution to the Problem of Division by Zero, in our Subsection 2.3 Type 0 Totalisation, employs an inchoate epsilon totalisation. He abandons arithmetic and defines division only at a meta level. This is logically satisfactory but arithmetically unsatisfactory. Suppes' 
third solution to the Problem of Division by Zero, in our Subsection 2.3 Type 0 Totalisation, is to define $x / 0=0$ for all real $x$. This is both logically and arithmetical satisfactory but Suppes says it is undesirable. Thus Suppes arrives at the position that, in 1957, there was no entirely satisfactory solution to the Problem of Division by Zero.

Let us explore the definition, $x / 0=0$ for all real $x$, in a little more detail.

We define that the lexical reciprocal, $r$, of zero is given by $r=1 / 0=$ $0^{-1}$. Let $r$ be an arbitrary real number. The field axiom for the multiplicative inverse is conditional: $a \times a^{-1}=1$ when $a \neq 0$. This allows $0^{-1}=r$. It is a theorem of the field axioms that $0 \times r=0$ for all real $r$. Hence all of the field axioms are satisfied for $0^{-1}=r$, where $r$ is an arbitrary real number. That is to say, any choice of $0^{-1}=r$ gives us a field. But now the product $0^{-1} \times a$ is fixed but arbitrary. Thus choosing any real number as the lexical reciprocal of zero produces some arbitrary products of the lexical reciprocal of zero. Hence, everywhere non-arbitrary products of the lexical reciprocal of zero arise only in totalisations of Extension Type at least 1.

Suppes' $x / 0=0$ for all real $x$ is a totalisation of Extension Type 0 and therefore has an arbitrary lexical reciprocal. We can see this directly. Choose $x=1$ then $x / 0=1 / 0=0$ but the choice $1 / 0=r=0$ is an arbitrary choice of $r$. Therefore the choice $x / 0=0$ is arbitrary.

Saitoh rediscovered the totalisation $x / 0=0$ for all real $x$. In a blog he dates this discovery to the 2nd February 2014. As we have seen, the choice $x / 0=0$ is arbitrary but Saitoh offers various external reasons to prefer this choice [10]. While we might be persuaded by Saitoh's arguments, they remain arbitrary. We could choose other functions and geometries that lead to different conclusions.

The choice of how to handle division by zero is likely to involve pragmatic considerations. As real analysis is of wide theoretical and practical importance, we are likely to want a totalised real arithmetic that works coherently with real analysis.

Real analysis adds two non-real symbols: $-\infty, \infty$. We can define an extended real analysis with any number of non-real symbols but if we want the symbols $-\infty, \infty$ to belong to our totalisation of real arithmetic then, trivially, we must use a totalisation of Extension Type at least 2 . However, not all totalisations of Extension Type 2 will suffice. The algebra of wheels [4] is Extension Type 2. It adds the symbols $\infty, \perp$ to an underlying number system, which we may take to be the real numbers. But wheels use a one-point compactification of the real number line, using the unsigned infinity, $\infty$, whence the compactification is a circle. But this circle is unorientable, hence the formerly real numbers lose the property of being ordered. There is no binary operation $a<b$ that applies to all elements $a, b$ of a wheel. In the absence of the operation less than, $<$, we cannot employ the epsilon-delta notation so no limits exist and there is no extended real analysis of a wheel, nor any analysis of a wheel. Wheels are inherently algebraic. By contrast, the transreal numbers, which are Extension Type 3, have a very well developed analysis [6] [7].

In terms of reception of Suppes 1957 as a source on division by zero, we could find some references to it, mentioning the fact that Suppes 1957 
covers this theme, but no work which proceeds with the challenge to survey options for dealing with division by zero and no specific options for dealing with division by zero seem to have originated from this work. However, we are unaware of any older reference to the totalisation of division by means of adopting $x / 0=0$. Suppes may have been original, among authors thinking in terms of modern mathematics and logic, in putting forward precisely this solution for the problem of division by zero. The rejection of allowing a modification of the underlying logic may have been convincing in 1957 but that position is certainly not shared universally. For instance in [8], a paper citing Suppes 1957, a modified logic, called positive free logic, is presented which achieves precisely the departure of classical firstorder logic needed to solve the difficulties as mentioned in Suppes 1957. Positive free logic does not prove $1 / 0=1 / 0$ but neither does it prove $1 / 0 \neq 1 / 0$ and consequently the kind of difficulties which are put forward, and which Suppes links in a plausible manner to the Goldbach conjecture, do not apply to positive free logic.

We are not specialists in the history of mathematics and computing but it may be helpful to give some historical context to Suppes 1957. Suppes, when looking at division by zero, was aware of the Riemann sphere and similar constructions which suggest an unsigned infinite value. But he was working from a background where: (i) floating point standards had yet to emerge, including the prescription that $1 / 0$ is given a singed infinite value; (ii) the theory of abstract datatypes and specifications thereof had yet to be developed; (iii) model theory and in particular model theory of fields was only beginning; (iv) there were no proof verification systems which might profit from the simplicity of typing achieved by adopting $1 / 0=0$; (v) logical investigations and proof systems concerning Kleene equality in partial algebra's were yet to come; and (vi) the very idea of theoretical computer science was nonexistent. Given such circumstances it is to be expected, in hindsight, that Suppes did not achieve a complete survey of possible approaches, while it can be admired that he was aware of the presence of the problem of division by zero, which will probably not be settled by means of a single universal solution.

\section{Conclusion}

The book "Introduction to Logic" by Patrick Suppes, 1957, is a pedagogical introduction to first-order predicate calculus with logical, mathematical, physical and philosophical examples. It is notable for considering five totalisations of real arithmetic. We classify totalisations by the number of non-real symbols they introduce. We compare the totalisations in Suppes' book to some modern approaches. We find that the Extension Type 0 totalisation, all real numbers divided by zero are equal to zero, was known to Suppes, but it leads to an arbitrary choice of the lexical reciprocal of zero. In fact all Extension Type 0 totalisations of real arithmetic have an arbitrary lexical reciprocal of zero but higher Extension Types may be non-arbitrary. If a totalisation of real arithmetic is to provide the symbols, $-\infty$ and $\infty$, of real analysis, it must be of Extension Type at least 2 . Wheels are an Extension Type 2 totalisation but they provide unordered 
numbers so cannot support any limits and, in particular, cannot totalise real analysis. Real analysis is totalised by the transreals, which are an Extension Type 3 totalisation. Interestingly, Suppes anticipated the use of a bottom element to totalise real arithmetic. Suppes arrived at the conclusion that there is no entirely satisfactory solution to the problem of division by zero.

\section{Acknowledgement}

We would like to thank Jonathan K. Cender for bringing the book Introduction to Logic to our attention and pointing out that Chapter 8 deals with totalisations of real arithmetic.

\section{References}

[1] J.A.D.W. Anderson, N. Völker, and A.A. Adams. Perspex machine viii: Axioms of transreal arithmetic. In Longin Jan Lateki, David M. Mount, and Angela Y. Wu, editors, Vision Geometry XV, volume 6499 of Proceedings of SPIE, pages 2.1-2.12, 2007. https://www. doi.org/10.1117/12.698153

[2] J.A.D.W. Anderson. Book review: The cult of pythagoras. Transmathematica, pages 1-2, 2020. https://doi.org/10.36285/tm. 37

[3] J.A. Bergstra. Division by zero: A survey of options. Transmathematica, pages 1-20, 25 June 2019. https://doi.org/10.36285/tm. v0i0.17

[4] J. Carlström. Wheels - on division by zero. Mathematical Structures in Computer Science, 14(1):143-184, 2004. https://doi.org/10. $1017 / \mathrm{S} 0960129503004110$

[5] T.S. dos Reis, W. Gomide, and J.A.D.W. Anderson. Construction of the transreal numbers and algebraic transfields. IAENG International Journal of Applied Mathematics, 46(1):11-23, 2016. http: //www. iaeng.org/IJAM/issues_v46/issue_1/IJAM_46_1_03.pdf

[6] T.S. dos Reis and J.A.D.W. Anderson. Transreal calculus. IAENG International Journal of Applied Mathematics, 45(1):51-63, 2015. http://www. iaeng.org/IJAM/issues_v45/issue_1/IJAM_45_ 1_06.pdf

[7] T.S. dos Reis and J.A.D.W. Anderson. Transreal limits and elementary functions. In Haeng Kon Kim; Mahyar A. Amouzegar; Sio long Ao, editor, Transactions on Engineering Technologies, pages 209-225, Dordrecht, 2015. Springer. https://link.springer.com/ chapter/10 . 1007\%2F978-94-017-7236-5_15

[8] R.D. Gumb and K. Lambert. Definitions in nonstrict positive free logic. Modern Logic, 7(1):25-55, 1997. https://projecteuclid. org/journals/review-of-modern-logic/volume-7/issue-1/ Definitions-in-nonstrict-positive-free-logic/rml/ 1204900340.full 
[9] A.I. Holub. Compiler Design in C. Prentice-Hall International, 1990. https://holub.com/goodies/compiler/compilerDesignInC.pdf

[10] H. Michiwaki, S. Saitoh, and M. Yamada. Reality of the division by zero z $/ 0=0$. International Journal of Applied Physics and Mathematics, 6(1):1-8, 2016. http://www.ijapm.org/show-63-504-1. html

[11] P. Suppes. Introduction to Logic. Van Nostrand Reinhold Company, 1957. http://web.mit.edu/gleitz/www/Introduction $\% 20$ to $\%$ 20Logic\%20-\%20P.\%20Suppes\%20(1957)\%20WW.pdf 